\title{
CHIỀU DÀI LÀN CHUYỂN TỐC TRÊN ĐƯờNG CAO TỐC
}

\author{
NGUYỄN TRỌNG SƠN HÀ \\ Truờng Đại học Công nghiệp thành phố Hồ Chí Minh
}

Tóm tắt. Để đạt được mục tiêu xe chạy đạt vận tốc thiết kế, êm thuận và an toàn cho người và phương tiện khi tham gia lưu thông vào và ra đường cao tốc, thì khi thiết kế bình đồ tuyến ngoài việc bảo đảm tầm nhìn trên đường, các tiêu chuẩn kỹ thuật đối với tuyến đường cao tốc trên bình đồ và trắc dọc, cần phải quan tâm đến chiều dài làn chuyển tốc. Nội dung bài báo đề cập phương pháp tính toán chiều dài tổng cộng của làn chuyển tốc theo Tiêu chuẩn Việt Nam TCVN 5729:2012 - Đường ô tô cao tốc - Yêu cầu thiết kế, so sánh kết quả với Tiêu chuẩn Việt Nam TCVN 4054:2005 Đường ô tô - Yêu cầu thiết kế, rút ra những kết luận cần thiết phục vụ cho công tác thiết kế các tuyến đường bộ trong tương lai, công tác cải tạo sửa chữa nâng cấp đường trên bình đồ.

Từ khóa. Chiều dài làn chuyển tốc, Đường cao tốc, Làn chuyển tốc, TCVN 5729:2012, TCVN 4054:2005.

\section{THE LENGTHS OF SPEED-CHANGE LANES OF HIGHWAY}

\begin{abstract}
In order to achieve the purpose of speed with the design, comfort and safety for people and vehicles when joining in and out of expressways, the technical standards not only pay attention for the highway on the planes and vertical tracking, but also pay attention to the speed-change lane. Content of the article refers to the method of calculating the total length of the speed change lane in accordance with Vietnam Standard TCVN 5729: 2012 - High-way designed requirements, comparison with TCVN 4054 : Roadways - designed requirements. This makes conclusions for the future design of high-ways and roads improvements and upgrades.
\end{abstract}

Keyword. The lengths of speed-change lanes, High-way, speed-change lanes, TCVN 5729:2012, TCVN 4054:2005.

\section{MỞ ĐẦU}

Đường ô tô cao tốc được xây dựng nhằm phục vụ giao thông có tốc độ cao, giao thông liên tục, đáp ứng lưu lượng và khả năng thông hành lớn. Đường cao tốc thường phục vụ nối liền giữa các đô thị lớn, giữa đô thị trung tâm với các trung tâm công nghiệp, bến cảng, nhà ga lớn, đô thị vệ tinh [3].

Để đạt được mục tiêu xe chạy đạt vận tốc thiết kế, êm thuận và an toàn cho người và phương tiện khi tham gia lưu thông vào và ra đường cao tốc, thì khi thiết kế tuyến ngoài việc bảo đảm tầm nhìn trên đường, các tiêu chuẩn kỹ thuật đối với tuyến đường cao tốc trên bình đồ và trắc dọc, cần phải quan tâm đến chiều dài làn chuyển tốc $[1,2,3,4,5]$. Làn chuyển tốc được bố trí ở các chỗ xe chuyển hướng vào các đường khác cấp. Bố trí làn tăng tốc khi xe từ đường có tốc độ thiết kế thấp vào các đường có tốc độ thiết kế cao. Ngược lại, bố trí làn giảm tốc [1]. Trong nội dung sâu đây sẽ trình bày cách thiết lập chiều dài tổng cộng của làn chuyển tốc theo Tiêu chuẩn Việt Nam TCVN 5729:2012 - Đường ô tô cao tốc - Yêu cầu thiết kế, so sánh kết quả với Tiêu chuẩn Việt Nam TCVN 4054:2005 Đường ô tô - Yêu cầu thiết kế, rút ra những kết luận cần thiết phục vụ cho công tác thiết kế các tuyến đường bộ trong tương lai, công tác cải tạo sửa chữa nâng cấp đường bộ trên bình đồ.

\section{CƠ SỞ TÍNH TOÁN CHIỀU DÀI LÀN CHUYỂN TỐC}

2.1. Theo TCVN 5729:2012 - Đường ô tô cao tốc - Yêu cầu thiết kế

2.1.1. Tiêu chuẩn kỹ thuật chủ yếu đối với tuyến đường cao tốc 
Các tiêu chuẩn kỹ thuật chủ yếu của các yếu tố hình học tuyến các cấp đường cao tốc trên bình đồ và mặt cắt dọc được quy định ở Bảng 1 .

Bảng 1. Tiêu chuẩn kỹ thuật chủ yếu đối với tuyến đường cao tốc

\begin{tabular}{|c|c|c|c|c|c|c|}
\hline STT & Tên chỉ tiêu & Đơn vị & \multicolumn{4}{|c|}{ Cấp đường } \\
\hline 1 & Tốc độ tính toán $\mathrm{V}_{\mathrm{tt}}$ & $\mathrm{km} / \mathrm{h}$ & 60 & 80 & 100 & 120 \\
\hline 2 & $\begin{array}{l}\text { Độ dốc siêu cao (hay độ nghiêng một } \\
\text { mái) lớn nhất } i_{S C} \text { không lớn hơn }\end{array}$ & $\%$ & 8 & 8 & 8 & 8 \\
\hline 3 & $\begin{array}{l}\text { Bán kính nhỏ nhất } \mathrm{R}_{\min } \text { tương ứng với } \\
\mathrm{i}_{\mathrm{SC}}=+8 \%, \mathrm{~m}\end{array}$ & $\mathrm{~m}$ & 140 & 240 & 450 & 650 \\
\hline 4 & $\begin{array}{l}\text { Bán kính nhỏ nhất thông thường tương } \\
\text { ứng với isC }=+5 \%\end{array}$ & $\mathrm{~m}$ & 250 & 450 & 650 & 1000 \\
\hline 5 & Bán kính tương ứng với $\mathrm{i}_{\mathrm{SC}}=+2 \%$ & $\mathrm{~m}$ & 700 & 1300 & 2000 & 3000 \\
\hline 6 & $\begin{array}{l}\text { Bán kính không cần cấu tạo nghiêng một } \\
\text { mái } i_{S C}=-2 \%\end{array}$ & $\mathrm{~m}$ & 1500 & 2500 & 4000 & 5500 \\
\hline 7 & $\begin{array}{l}\text { Chiều dài đường cong chuyển tiếp ứng } \\
\text { với } R_{\min }\end{array}$ & $\mathrm{m}$ & 150 & 170 & 210 & 210 \\
\hline 8 & $\begin{array}{l}\text { Chiều dài đường cong chuyển tiếp ứng } \\
\text { với bán kính nhỏ nhất thông thường }\end{array}$ & $\mathrm{m}$ & 90 & 140 & 150 & 150 \\
\hline 9 & $\begin{array}{l}\text { Chiều dài đường cong chuyển tiếp ứng } \\
\text { với bán kính có trị số trong ngoặc }\end{array}$ & $\mathrm{m}$ & $\begin{array}{c}50 \\
(450) \\
\end{array}$ & $\begin{array}{c}75 \\
(675) \\
\end{array}$ & $\begin{array}{r}100 \\
(900) \\
\end{array}$ & $\begin{array}{c}125 \\
(1125) \\
\end{array}$ \\
\hline 10 & Chiều dài hãm xe (hay tầm nhìn dừng xe) & $\mathrm{m}$ & 75 & 110 & 160 & 230 \\
\hline 11 & Độ dốc dọc lên dốc lớn nhất & $\%$ & 6 & 6 & 5 & 4 \\
\hline 12 & Độ dốc dọc xuống dốc lớn nhất & $\%$ & 6 & 6 & 5,5 & 5,5 \\
\hline 13 & Bán kính đường cong đứng lồi tối thiểu & $\mathrm{m}$ & 1500 & 3000 & 6000 & 12000 \\
\hline 14 & $\begin{array}{l}\text { Bán kính đường cong đứng lõm tối thiểu, } \\
\text { m }\end{array}$ & $\mathrm{m}$ & 1000 & 2000 & 3000 & 5000 \\
\hline
\end{tabular}

CHÚ THÍCH Bảng 1: Tốc độ tính toán $\mathrm{V}_{\mathrm{tt}}$ ở đây được hiểu là tốc độ dùng để tính toán xác định các tiêu chuẩn giới hạn đối với các yếu tố hình học được bố trí ở một số chỗ cá biệt trên tuyến đường cao tốc.

\subsubsection{Chỗ giao nhau trên đường cao tốc và các chỗ ra, vào đường cao tốc}

Khi thiết kế tuyến cần phải bảo đảm tầm nhìn trên đường, các tiêu chuẩn kỹ thuật đối với tuyến đường cao tốc trên bình đồ và trắc dọc, thiết kế chỗ giao nhau trên đường và các chỗ ra, vào đường cao tốc. Có thể bố trí chỗ ra từ đường cao tốc (chỗ tách dòng) theo hai cách bố trí song song hoặc nối trực tiếp như ở Hình 1. Đối với chỗ vào đường cao tốc (chỗ nhập dòng) chỉ nên bố trí theo cách song song (Hình 1.c) với toàn bộ chiều dài đoạn tăng tốc được đặt cạnh phần xe chạy của đường cao tốc (mở rộng phần lề) hoặc nếu chiều dài đoạn tăng tốc theo tính toán là quá dài thì tối thiểu phải có $100 \mathrm{~m}$ của nó được đặt cạnh phần xe chạy của đường cao tốc.

a)

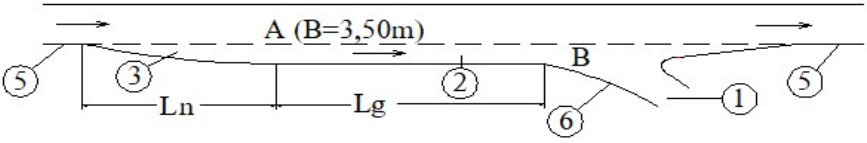

b)

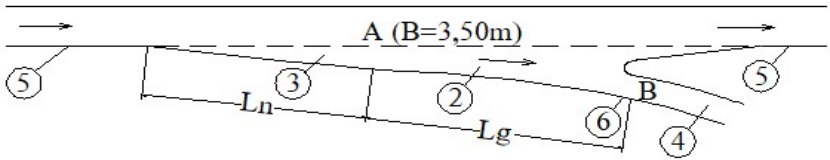

c)

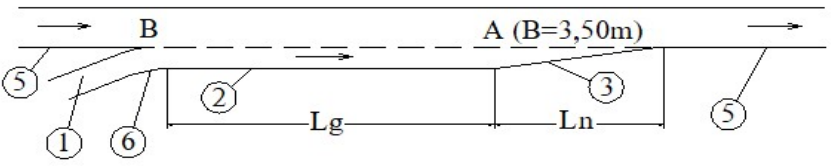

Hinh 1. Các giải pháp bố trí chỗ ra, vào đường cao tốc 


\section{CHÚ THÍCH Hình 1:}

$\mathrm{a}$ - Chỗ ra kiểu bố trí song song;

$\mathrm{b}$ - Chỗ ra kiểu nối trực tiếp;

$\mathrm{c}$ - Chỗ vào đường cao tốc kiểu bố trí song song; Bán kính cong tại $\mathrm{B}$ : $\mathrm{r}=0,6 \mathrm{~m} \div 1,0 \mathrm{~m}$.

1 - Đường nhánh;

2 - Đoạn chuyển tốc $\mathrm{L}_{\mathrm{g}}$ (giảm tốc với trường hợp Hình a và b; và tăng tốc với trường hợp Hình $\mathrm{c}$ );

3 - Đoạn chuyển làn hình nêm Ln;

4 - Đường nhánh kiêm luôn chức năng chuyển làn và chuyển tốc;

5 - Mép phần xe chạy (không kể lề) của đường cao tốc;

6 - Đoạn chuyển tiếp clothoide;

Ln - Đoạn chuyển làn hình nêm.

Điểm $\mathrm{A}$ là tại đó đoạn chuyển làn hình nêm có chiều rộng đủ $3,5 \mathrm{~m}$.

Điểm $\mathrm{B}$ được xác định là xe có tốc độ tính toán trên đường nhánh.

\subsubsection{Chiều dài làn chuyển tốc}

a) Chiều dài đoạn hình nêm:

Chiều dài tối thiểu đoạn chuyển làn hình nêm (kể cả trường hợp rẽ ra hoặc vào đường cao tốc) được xác định tùy thuộc cấp đường cao tốc như ở Bảng 2.

Bảng 2. Chiều dài tối thiểu đoạn chuyển làn hình nêm $\mathrm{L}_{\mathrm{n}}$ (tách hoặc nhập dòng). (Đơn vị tính bằng $m$.)

\begin{tabular}{|c|c|c|c|c|}
\hline Cấp đường cao tốc & 120 & 100 & 80 & 60 \\
\hline $\mathrm{L}_{\mathrm{n}}$ & 75 & 60 & 50 & 40 \\
\hline
\end{tabular}

b) Chiều dài đoạn chuyển tốc:

Đoạn giảm hoặc tăng tốc được kể từ điểm $\mathrm{A}$ (cuối hoặc đầu đoạn hình nêm) trên hình 1 và chiều dài $\mathrm{L}_{\mathrm{g}}$ (tính bằng mét) của chúng được xác định theo công thức:

$$
L_{g}=\frac{V_{A}^{2}-V_{B}^{2}}{26 . a}
$$

Trong đó:

- $\mathrm{V}_{\mathrm{A}}$ là tốc độ xe chạy tại điểm $\mathrm{A}$ (cuối đoạn chuyển làn hình nêm) trên hình 1 , tính bằng $\mathrm{km} / \mathrm{h}$;

Trị số $\mathrm{V}_{\mathrm{A}}$ được quy định theo cấp đường cao tốc ở Bảng 3 .

- $a$ là gia tốc tăng hoặc giảm tốc, tính bằng $\mathrm{m} / \mathrm{s}^{2}$;

Khi xác định chiều dài đoạn giảm tốc lấy $\mathrm{a}=2,5 \mathrm{~m} / \mathrm{s}^{2}$, còn khi xác định chiều dài của đoạn tăng tốc lấy $\mathrm{a}=1,0 \mathrm{~m} / \mathrm{s}^{2}$.

- $\mathrm{V}_{\mathrm{B}}$ là tốc độ ở cuối đoạn giảm tốc hay đầu đoạn tăng tốc, tính bằng $\mathrm{km} / \mathrm{h}$;

Trị số $\mathrm{V}_{\mathrm{B}}$ được xác định tùy thuộc tốc độ tính toán trên đường nhánh hoặc tùy thuộc các yếu tố hình học thực tế được áp dụng trên đường nhánh khi thiết kế cụ thể ở sau đoạn giảm tốc hoặc trước đoạn tăng tốc.

Bảng 3. Trị số tốc độ $\mathrm{V}_{\mathrm{A}}$ ở đầu đoạn giảm tốc hoặc cuối đoạn tăng tốc. (Đơn vị tính bằng $\mathrm{km} / \mathrm{h}$.)

\begin{tabular}{|c|c|c|c|c|}
\hline Cấp đường cao tốc & 120 & 100 & 80 & 60 \\
\hline $\mathrm{V}_{\mathrm{A}}$ & 80 & 70 & 60 & 50 \\
\hline
\end{tabular}

c) Chiều dài làn chuyển tốc:

Trong mọi trường hợp, tùy thuộc cấp đường cao tốc, chiều dài toàn bộ làn chuyển tốc $\mathrm{L}_{\mathrm{v}}$ là tổng chiều dài đoạn chuyển làn (hình nêm) cộng với chiều dài đoạn chuyển tốc (giảm hoặc tăng tốc):

$$
L_{v}=L_{n}+L_{g} \quad(2)
$$

$\mathrm{L}_{\mathrm{v}}$ nên lớn hơn trị số cho ở Bảng 4 , và sẽ nhân thêm với hệ số hiệu chỉnh nếu làn chuyển tốc xuống dốc hoặc lên dốc [1].

Bảng 4. Trị số tối thiểu áp dụng cho tổng chiều dài đoạn chuyển làn cộng với đoạn chuyển tốc. (Đơn vị tính bằng $m$.)

\begin{tabular}{|l|c|c|c|c|}
\hline Cấp đường cao tốc & $\mathbf{1 2 0}$ & $\mathbf{1 0 0}$ & $\mathbf{8 0}$ & $\mathbf{6 0}$ \\
\hline Chiều dài tối thiểu ở chỗ ra (giảm tốc) 1 làn xe, $\mathrm{m}$ & $100(150)$ & $90(130)$ & $80(110)$ & $70(90)$ \\
\hline Chiều dài tối thiểu ở chỗ vào (tăng tốc) 1 làn xe, $\mathrm{m}$ & $200(300)$ & $180(260)$ & $160(220)$ & $120(160)$ \\
\hline
\end{tabular}

CHÚ THÍCH Bảng 4: Trị số trong ngoặc tương ứng với trường hợp đoạn chuyển tốc gồm 2 làn xe. 


\subsection{Theo TCVN 4054:2005 - Đường ô tô - Yêu cầu thiết kế}

TCVN 4054:2005 cũng qui định làn giảm tốc cấu tạo theo kiểu song song hoặc theo kiểu nối trực tiếp (xem Hình 1.a và 1.b), làn tăng tốc cấu tạo theo kiểu song song (theo Hình 1.c).

Làn chuyển tốc có chiều rộng là $3,50 \mathrm{~m}$. Chiều dài đoạn hình nêm tối thiểu dài $35 \mathrm{~m}$ (mở rộng $1 \mathrm{~m}$ trên chiều dài $10 \mathrm{~m}$ ). Chiều dài đoạn chuyển tốc tính theo gia tốc dương là $1 \mathrm{~m} / \mathrm{s}^{2}$, gia tốc âm là $2 \mathrm{~m} / \mathrm{s}^{2}$; so sánh với TCVN 5729:2012 thì ở đây có sự sai khác, tức gia tốc âm là $2,5 \mathrm{~m} / \mathrm{s}^{2}$. Chiều dài đoạn giảm tốc lấy không dưới $30 \mathrm{~m}$, chiều dài đoạn tăng tốc không dưới $120 \mathrm{~m}$ [2]. Do có sự chồng lấn về vận tốc thiết kế giữa TCVN 4054:2005 và TCVN 5729:2012 nên vấn đề này cần được quan tâm khi thiết kế tuyến đường có $\mathrm{V}_{\mathrm{TK}}$ lớn theo TCVN 4054:2005.

\section{3. ÁP DUUNG TÍNH TOÁN CHIỀU DÀI LÀN CHUYỄN TỐC}

Theo Tiêu chuẩn xây dựng Việt Nam, TCXDVN 104:2007 Đường đô thị - Yêu cầu thiết kế, Bộ Xây dựng 2007, xác định tốc độ thiết kế ở nhánh nối có thể tham khảo Bảng 5 [3].

Bảng 5. Lựa chọn tốc độ thiết kế trên nhánh nối

\begin{tabular}{|c|c|c|c|}
\hline \multirow{2}{*}{$\begin{array}{l}\text { VK } \\
\text { trên đường giao } \\
(\mathrm{km} / \mathrm{h})\end{array}$} & Mức cao & Mức trung bình & Mớc độ thiết kế trên nháp \\
\cline { 2 - 4 } & $80-70$ & 60 & 50 \\
70 & 70 & 55 & 45 \\
80 & $70-60$ & 50 & 40 \\
60 & 50 & 40 & 30 \\
50 & 40 & 30 & 25 \\
\hline
\end{tabular}

\section{CHÚ THÍCH Bảng 5:}

- Nhánh nối rẽ phải thường áp dụng tốc độ ở mức trung bình tới cao.

- Nhánh nối gián tiếp thường áp dụng tốc độ ở mức thấp.

- Nhánh nối bán trực tiếp thường áp dụng tốc độ ở mức trung bình tới cao.

Nếu chiều dài nhánh nối ngắn, làn xe đơn nên lấy $\leq 60 \mathrm{~km} / \mathrm{h}$.

Dựa trên Bảng 5 , tác giả lựa chọn tốc độ trung bình của nhánh nối $\mathrm{V}_{\mathrm{B}}=40 \mathrm{~km} / \mathrm{h}$ để áp dụng tính toán. Vận dụng công thức (1) và (2), các dữ liệu tại các Bảng $2,3,4$ để tính toán chiều dài làn chuyển tốc cho đường có 1 làn xe trên đường bằng. Kết quả được ghi ở Bảng 6 và Bảng 7 .

Bảng 6. Kết quả tính toán Lv theo TCVN 5729:2012

\begin{tabular}{|c|c|c|c|c|c|}
\hline STT & Cấp đường cao tốc & 120 & 100 & 80 & 60 \\
\hline 1 & Chiều dài tối thiểu ở chỗ ra (giảm tốc) 1 làn xe, $m$ & 100 & 90 & 80 & 70 \\
\hline 2 & Chiều dài tối thiểu ở chỗ vào (tăng tốc) 1 làn xe, $m$ & 200 & 180 & 160 & 120 \\
\hline 3 & $\begin{array}{l}\text { Chiều dài tối thiểu đoạn chuyển làn hình nêm } \mathrm{L}_{\mathrm{n}} \text { (tách } \\
\text { hoặc nhập dòng), m. }\end{array}$ & 75 & 60 & 50 & 40 \\
\hline 4 & $\begin{array}{l}\text { Chiều dài tính toán tối thiểu đoạn tăng tốc } \operatorname{Lg}(+) \\
\text { (nhập dòng), } \mathrm{m} \text {. }\end{array}$ & 185 & 127 & 77 & 35 \\
\hline 5 & $\begin{array}{l}\text { Chiều dài tính toán tối thiểu đoạn giảm tốc } \operatorname{Lg}(-) \text { (tách } \\
\text { dòng), m. }\end{array}$ & 74 & 51 & 31 & 14 \\
\hline 6 & Chiều dài tính toán tổng cộng $\mathrm{L}_{\mathrm{v}}$ chỗ vào, $\mathrm{m}$. & 260 & 187 & 127 & 75 \\
\hline 7 & Chọn chiều dài làn chuyển tốc chỗ vào: & $\begin{array}{l}\text { Theo tính } \\
\text { toán }\end{array}$ & $\begin{array}{l}\text { Theo tính } \\
\text { toán }\end{array}$ & $\begin{array}{c}\text { Theo } \\
\text { TCVN } \\
5729: 2012\end{array}$ & $\begin{array}{c}\text { Theo } \\
\text { TCVN } \\
5729: 2012\end{array}$ \\
\hline 8 & $\begin{array}{l}\text { Chiều dài tính toán tổng cộng } \mathrm{L}_{\mathrm{v}} \text { chỗ } \mathrm{ra}, \mathrm{m} \text {, theo } \\
\text { TCVN 5729:2012 }\end{array}$ & 149 & 111 & 81 & 54 \\
\hline 9 & Chọn chiều dài làn chuyển tốc chỗ ra: & $\begin{array}{l}\text { Theo tính } \\
\text { toán }\end{array}$ & $\begin{array}{l}\text { Theo tính } \\
\text { toán }\end{array}$ & $\begin{array}{l}\text { Theo tính } \\
\text { toán }\end{array}$ & $\begin{array}{c}\text { Theo } \\
\text { TCVN } \\
5729: 2012\end{array}$ \\
\hline
\end{tabular}


Bảng 7. Kết quả tính toán Lv theo TCVN 4054:2005

\begin{tabular}{|c|c|c|c|c|c|}
\hline STT & Tốc độ thiết kế, $\mathrm{V}_{\mathrm{TK}}, \mathrm{km} / \mathrm{h}$ & 120 & 100 & 80 & 60 \\
\hline 1 & $\begin{array}{l}\text { Chiều dài tối thiểu đoạn chuyển làn hình nêm } \mathrm{L}_{\mathrm{n}} \text { (tách } \\
\text { hoặc nhập dòng), } \mathrm{m} \text {. }\end{array}$ & 35 & 35 & 35 & 35 \\
\hline 2 & Chiều dài tối thiểu đoạn chuyển tốc $\mathrm{L}_{\mathrm{g}}$ (nhập dòng), $\mathrm{m}$. & 120 & 120 & 120 & 120 \\
\hline 3 & Chiều dài tối thiểu đoạn chuyển tốc $\mathrm{L}_{\mathrm{g}}$ (tách dòng), m. & 30 & 30 & 30 & 30 \\
\hline 4 & Chiều dài tổng cộng (nhập dòng) $\mathrm{L}_{\mathrm{v}}, \mathrm{m}$. & 155 & 155 & 155 & 155 \\
\hline 5 & Chiều dài tống cộng (tách dòng) $\mathrm{L}_{\mathrm{v}}, \mathrm{m}$. & 65 & 65 & 65 & 65 \\
\hline 6 & Chọn chiều dài làn chuyển tốc: & $\begin{array}{c}\text { Theo } \\
\text { TCVN } \\
5729: 2012\end{array}$ & $\begin{array}{c}\text { Theo } \\
\text { TCVN } \\
\text { 5729:2012 }\end{array}$ & $\begin{array}{c}\text { Theo } \\
\text { TCVN } \\
\text { 5729:2012 }\end{array}$ & $\begin{array}{c}\text { Theo } \\
\text { TCVN } \\
\text { 4054:2005 }\end{array}$ \\
\hline
\end{tabular}

Nhận xét về các kết quả tính toán $\mathrm{L}_{\mathrm{v}}$ :

- Theo TCVN 5729:2012: Với điều kiện nhánh nối cụ thể như trên thì chiều dài làn chuyển tốc nhập dòng với cấp đường 120,100 và chiều dài làn chuyển tốc tách dòng với cấp đường $120,100,80$ nên lấy theo kết quả tính toán.

- Theo TCVN 4054:2005 thì kết quả tính toán $\mathrm{L}_{\mathrm{v}}$ cho thấy chiều dài làn chuyển tốc nhập dòng và tách dòng với các tốc độ thiết kế $120,100,80$ đều nhỏ hơn so với kết quả tính toán theo TCVN 5729:2012.

\section{KẾT LUẬn}

$>$ Chiều dài làn chuyển tốc có sự khác nhau giữa các kết quả tính toán theo TCVN 5729:2012 và TCVN 4054:2005. Chiều dài làn chuyển tốc theo TCVN 5729:2012 lớn hơn do qui định của Yêu cầu thiết kế đường cao tốc cao hơn Yêu cầu thiết kế đường ô tô. Do có sự chồng lấn về vận tốc thiết kế giữa TCVN 4054:2005 và TCVN 5729:2012 nên vấn đề này cần được quan tâm khi thiết kế tuyến đường có vận tốc thiết kế lớn (từ $80 \mathrm{~km} / \mathrm{h}$ ) theo TCVN 4054:2005.

$>$ Trường hợp thiết kế chiều dài làn chuyển tốc trên đường cao tốc nên tính toán với các nút giao cụ thể theo qui định của TCVN 5729:2012 - Đường ô tô cao tốc - Yêu cầu thiết kế.

$>$ Khi thiết kế chiều dài làn chuyển tốc trên đường ôtô tuân thủ qui định của TCVN 4054:2005 Đường ô tô - Yêu cầu thiết kế; Tuy nhiên ở cấp đường cao hơn (từ $80 \mathrm{~km} / \mathrm{h}$ ) nên tuân thủ TCVN 5729:2012 - Đường ô tô cao tốc - Yêu cầu thiết kế.

\section{TÀI LIỆU THAM KHẢO}

[1] Tiêu chuẩn Việt Nam, TCVN 5729:2012 - Đường ô tô cao tốc - Yêu cầu thiết kế, Hà Nội 2012.

[2] Tiêu chuẩn Việt Nam, TCVN 4054:2005 Đường ô tô - Yêu cầu thiết kế, Hà Nội 2005.

[3] Tiêu chuẩn xây dựng Việt Nam, TCXDVN 104:2007 Đường đô thị - Yêu cầu thiết kế, Bộ Xây dựng 2007.

[4] Tiêu chuẩn ngành, 22 TCN 273-01 Tiêu chuẩn thiết kế đường ô tô, Bộ Giao thông Vận tải 2001.

[5] Tiêu chuẩn ngành, 22 TCN 272-05 Tiêu chuẩn thiết kế cầu, Bộ Giao thông Vận tải 2005.

Ngày nhận bài: 29/09/2017

Ngày chấp nhận đăng: 31/12/2017 